Special issue of the 2nd International Conference on Computational and Experimental Science and Engineering (ICCESEN 2015)

\title{
A Novel Application of the Quartz Crystal Microbalance for Determining the Rheological Properties of the Highly Viscous Liquids
}

\author{
A.A. BARZINJY ${ }^{a}$ AND M.M. ZANKANA ${ }^{b, *}$ \\ ${ }^{a}$ Department of Physics, College of Education, University of Salahaddin, Erbil, Iraq \\ ${ }^{b}$ Department of Chemistry, University of Garmian, Sulaimani, Iraq
}

\begin{abstract}
Traditional methods such as rotational viscometer and other viscometer methods are limited in their ability to investigate rheological properties of high viscous liquids. This study introduces a new approach to overcome these obstacles. Deep eutectic solvents suffer from high viscosity and they are relatively expensive. Therefore, there is a requirement to both reduce the volumes of samples required and increase the accuracy of the technique at high viscosities. Quartz crystal microbalance techniques have been applied because they provide quick recording, the potential for collecting a large amount of data in a short time. Furthermore, the quartz crystal microbalance microviscometer has the advantage over most other methods of viscometry not in its precision of absolute viscosity measurement but in the very small amount of sample liquid needed for investigation. To test the precision of quartz crystal microbalance a comparison must be made between readings obtained with a rotational viscometer set up alongside. In this investigation a series of deep eutectic solvents with various viscosities and physical characteristics have been tested. The influence of the addition of water to the liquids was also assessed.
\end{abstract}

DOI: 10.12693/APhysPolA.130.239

PACS/topics: 83.85.Cg, 83.85.Jn

\section{Introduction}

Once the quartz crystal microbalance (QCM) was first introduced in 1959 [1], it characterized a most important step forward in our aptitude to weigh matter. This method extended the sensitivity by two or three orders of magnitude, into the sub ng range [2]. Nevertheless, to use the QCM as a mass sensor one must be certain that the measured mass is strictly attached to its surface. When dealing with liquids it is significant that throughout the process studied there is no change in the roughness of this surface and that the viscosity and density of the nearby medium remain constant. Only under these circumstances will the frequency shift, $\Delta f$, be relative to the change in mass, $\Delta m$, expressed in units of mass per surface area,

$$
\Delta f=-C_{m} \Delta m
$$

where the constant, $C_{m}$, is determined by the properties of the QCM [1]. Moreover, the negative sign in Eq. (1) shows that addition of mass to the resonator fallouts in a decrease in its resonant frequency and vice versa.

The sensitivity of the QCM is obvious as a reduction of oscillating frequency relative to the loading. In the case of solid thin films the frequency change $\Delta f$ is accompanying the film mass. In the case of liquids this is associated with the liquid viscosity [3]. A new area of this investigation had been, fundamentally, explored and was supposed to be applicable to this solvent viscosity

*corresponding author; e-mail: masoudzankanachemist@gmail.com study. This area was the determination of liquid viscosity by the application of a small amount of sample to one electrode of a freely oscillating flat crystal [4-8].

Recently the QCM has been utilized to analyze viscous properties of liquids. Kanazawa and Gordon [3] established a correlation between $\Delta f$ and kinematic viscosity based on a physical model which combines the shear wave in the quartz to the damped shear wave in the fluid

$$
\Delta f=-f_{0}^{3 / 2} \sqrt{\frac{\eta_{l} \rho_{l}}{\pi \mu_{q} \rho_{q}}},
$$

where $\Delta f$ is the essential frequency (of the bare crystal), $\eta_{l}$ and $\rho_{l}$ are the absolute viscosity and density of the liquid in that order. Ward and Buttry [9] showed that the current QCM arrangements are not satisfactory for commercial usage.

This study presents investigation accomplished in the field of the QCM and more precisely, liquid viscosity measurement by means of the QCM technique. This novel technique will be helpful, as it will allow the classification of properties of a fluid when only very small quantities are produced. This is valuable when the properties of new fluids are being determined, as commonly new fluids will be produced in small quantities.

This study focused on deep eutectic solvents (DESs) which are non-volatile liquid and therefore a new area of research. DESs are now broadly known as a novel class of ionic liquid (IL) analogues since they share many features and properties with ILs. The expressions DES and IL have been utilized interchangeably in the literature however it is essential to mention that these are actually two dissimilar types of solvent. DESs are systems designed from a eutectic mixture of Lewis or Brønsted acids 
and bases which can comprise a range of anionic and/or cationic species; in contrast, ILs are formed from systems composed mainly of one type of discrete anion and cation. In general, ILs have higher viscosities than the other molten salts and aqueous solutions [10]. The consequence of this problem was the invention of DES with lower viscosities which contributes to Van der Waals force between molecules and alters their tendency to form hydrogen bonds.

The calculated viscosities from the less widely known technique, QCM, were compared in situ with results from a conventional rotational viscometer. In this study a series of DESs, namely ethaline, glyceline, reline, and maline with various viscosities and physical characteristics have been tested. It was our aim to investigate the influence of the addition of water to these solvents as well.

\section{Experimental}

This investigation assessed four DESs: ethaline, glyceline, reline, and maline. In all mentioned DESs choline chloride was used as the quaternary ammonium salt with a variety of hydrogen bond donors (HBDs). For ethaline preparation choline chloride $\left(\mathrm{C}_{5} \mathrm{H}_{14} \mathrm{ClNO}\right)$ was added to ethylene glycol $\left(\mathrm{C}_{2} \mathrm{H}_{6} \mathrm{O}_{2}\right)$ in a 1:2 molar ratio. The resulting mixture was continually stirred with heating $\left(\mathrm{ca} .45-50^{\circ} \mathrm{C}\right)$, until a homogeneous liquid was formed. Glyceline was synthesized by the same method as ethaline except that glycerol $\left(\mathrm{C}_{3} \mathrm{H}_{8} \mathrm{O}_{3}\right)$ was added in place of the ethylene glycol. The ratio was the same as for ethaline, a 1:2 molar ratio of choline chloride to glycerol. The preparation of reline was the same as the preparation of ethaline except for the fact that urea $\left(\mathrm{CH}_{4} \mathrm{~N}_{2} \mathrm{O}\right)$ was substituted in place of the ethylene glycol. This process maintained a 1:2 molar ratio of choline chloride to urea. Maline was prepared in the same manor retaining the use of choline chloride as the quaternary ammonium salt, however in this instance it was mixed with malonic acid $\left(\mathrm{C}_{3} \mathrm{H}_{4} \mathrm{O}_{4}\right)$ in a 1:1 molar ratio.

All QCM readings were acquired using an Agilent technologies E5061A $(300 \mathrm{kHz}-1.5 \mathrm{GHz})$ ENA Series Network Analyzer measuring impedance against frequency of a $10 \mathrm{MHz}$ crystal. Two voltmeters were attached to the network analyzer in order to act as reference points as well as to record the output of the attached thermocouple which was used.

In order to measure the temperature accurately during the collection of data from the QCM an Omega brand $J$ Type thermocouple was utilized. The thermocouple was utilized to obtain accurate temperature readings. The thermocouple is composed of two metals which were connected on one side. A voltage was observed when the temperature at the connection area of the two metals increased or decreased. This voltage could be used to infer the solution temperature.

\section{Theory}

Isaac Newton defined viscosity by considering the model (Fig. 1). Two parallel flat areas of fluid of the same size $(A)$ are separated by a distance $(\mathrm{d} x)$ and are moving in the same direction at different velocities $\left(V_{1}\right)$ and $\left(V_{2}\right)$. Newton assumed that the force $(F)$ required maintaining this difference in speed was proportional to the difference in speed through the liquid. This is expressed as Eq. (3) where $\eta$ is a constant for a given material called its viscosity

$$
\frac{F}{A}=\eta \frac{\mathrm{d} v}{\mathrm{~d} x} .
$$

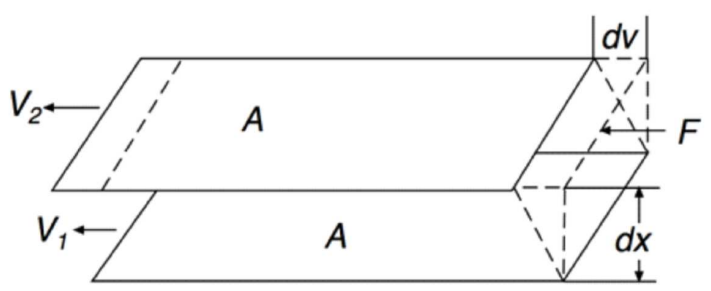

Fig. 1. Isaac Newton's model of viscosity.

The velocity gradient $(\mathrm{d} v / \mathrm{d} x)$ is a measure of the changing speed at which the layers move with respect to each other. It describes the shearing the liquid experiences i.e. shear rate. The term $F / A$ indicates the force per unit area required to produce the shearing action. Using these simplified terms, viscosity can be mathematically defined by (4):

$$
\text { viscosity }=\frac{\text { shear stress }}{\text { shear rate }} \text {. }
$$

Newton assumed that all materials have, at a given temperature, a viscosity that is independent of the shear rate. In other words applying twice the force would move the fluid twice as fast. This however only applies to fluids which show Newtonian behavior. Accordingly, Newtonian behavior can be divided into two types:

1. Newtonian fluids: A Newtonian fluid shows a linear relationship between shear stress and shear rate. For a Newtonian fluid viscosity remains constant independent of shear rate at a given temperature. A typical example of a Newtonian fluid is water, milk and other common chemical reagents, such as acetone, dichloromethane (DCM) and acetonitrile.

2. Non-Newtonian fluids: A non-Newtonian fluid is broadly defined as one for which the relationship between shear stress and shear rate is not constant. When the shear rate is varied, the shear stress does not vary in the same proportion as this change. An example would be blood, as the viscosity changes with rate of blood flow [11]. The viscosity of such fluids will therefore change as the shear rate is varied. Therefore the experimental parameters of the viscometer, spindle and speed all have an effect on the measured viscosity of a non-Newtonian fluid.

During in situ experiments, one side of the crystal is exposed to air and the other side is exposed to the solution. The resonant frequency of the crystal will be altered when it is in contact with a fluid. This change in 
frequency can be used to calculate the viscosity of the liquid that the crystal is exposed to. By rearranging Eq. (2), the viscosity of a liquid can be calculated as

$$
\eta=\frac{(\Delta f)^{2}}{f_{0}^{3} \rho_{\mathrm{L}}} \pi \rho_{q} \mu_{q} .
$$

The resonant frequency corresponds to the frequency of the peak at maximum height. When in contact with viscous fluids, the frequency of the crystal will decrease as the viscosity increases, resulting in a large $\Delta f$ for highly viscous fluids. The QCM method will also allow faster and more accurate data to be collected during an experiment, when compared to a rotational viscometer and other techniques. Viscosity values determined using a rotational viscometer are affected by the \%torque that the machine experiences at any given point during an experiment. When varying the temperature of a fluid throughout an experiment the \%torque also varies, this means that during the experiment the accuracy of the recorded viscosity constantly changes. In addition to this, the experimental setup, such as spindle size and speed, must be varied according to the viscosity of the fluid when using the rotational viscometer. Experimental factors such as these do not apply to the QCM; it can be used instantly for all fluids without altering any experimental settings.

\section{Results and discussion}

In order to obtain data to calculate the viscosity of the DES liquid the Kanazawa-Gorden equation was applied. One of the parameters required for this calculation is density. A KRUSS Tensiometer K9 was used to measure density. Furthermore, the measurements were carried out at various temperatures.

\subsection{Viscosity before adding water}

The viscosity of ethaline was measured using the rotational viscometer and QCM concurrently, and was shown to vary with temperature. At high temperatures the viscosity is at a minimum and at low temperatures the viscosity is at a maximum. It can be seen that from Fig. 2a ethaline has the lowest viscosity being exactly at the bottom of the curves. It has an approximately linear shape in comparison to the other fluids. This liquid has a viscosity of $13.8 \mathrm{cP}$ at $348 \mathrm{~K}$ and reaches $37.34 \mathrm{cP}$ at temperature of $303 \mathrm{~K}$. Whilst, other DES such as glyceline, reline, and maline start at higher levels of viscosity, 34.55, 41.39 , and $150.37 \mathrm{cP}$, respectively at $348 \mathrm{~K}$.

On the other hand, at $303 \mathrm{~K}$, similar phenomena have been observed. All DES viscosities started to increase gradually with decrease of the temperature except for ethaline. It is difficult to observe the variation in the viscosity of this solution, when it is compared with glyceline, reline, and maline only a small range of viscosities can be seen. At $303 \mathrm{~K}$ ethaline has a viscosity of $37.64 \mathrm{cP}$, which was considerably lower than the other three DES which had viscosities of $256.15,568.68$, and $1004.19 \mathrm{cP}$.

For ethaline, the viscosity ranges from $8.8 \mathrm{cP}$ at $85^{\circ} \mathrm{C}$ to a maximum of $52.9 \mathrm{cP}$ at room temperature $\left(25^{\circ} \mathrm{C}\right)$

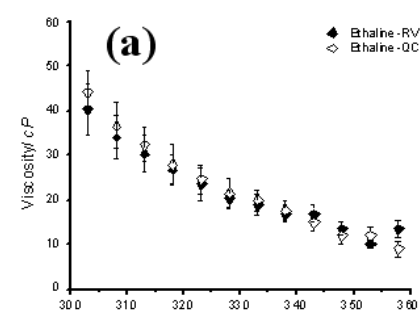

(b)
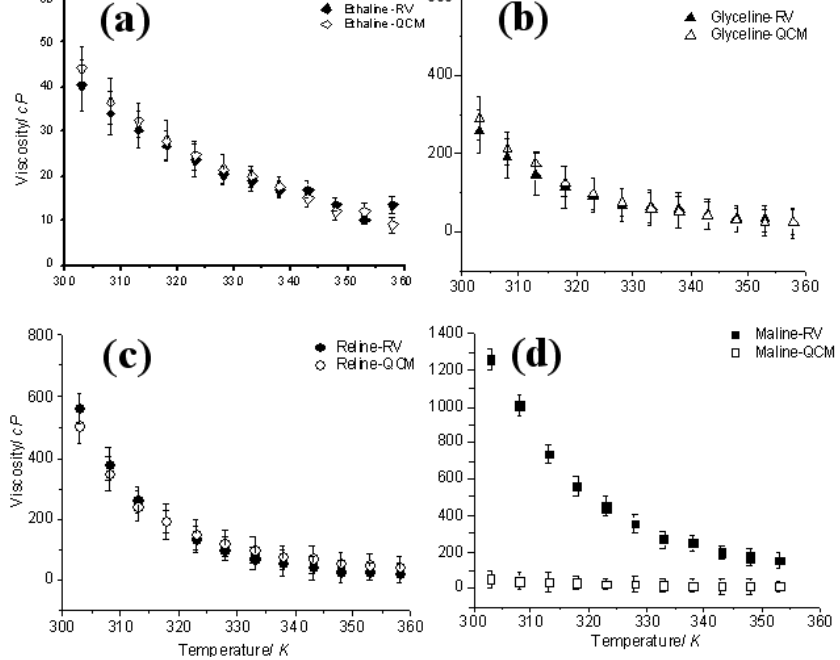

Fig. 2. Viscosity of (a) ethaline, (b) glyceline, (c) reline, and (d) maline calculated from QCM frequency change and by rotational viscometer (RV) as a function of temperature.

when calculated from the QCM data using the Kanazawa and Gordon equation [3]. From the rotational viscosity data, the viscosity ranges between $13.4 \mathrm{cP}$ at $85^{\circ} \mathrm{C}$ to $46.9 \mathrm{cP}$ at $25^{\circ} \mathrm{C}$. Both the methods produced similar results at low temperatures, with the viscosity differing by only $5 \mathrm{cP}$ at $25^{\circ} \mathrm{C}$. There is a crossover point with the two curves, at $40^{\circ} \mathrm{C}$, where the difference in values begins to increase, towards a maximum deviation in viscosity of $7 \mathrm{cP}$ at $80^{\circ} \mathrm{C}$. The literature $[12]$ reports the viscosity of ethaline as being $36 \mathrm{cP}$ at $20^{\circ} \mathrm{C}$, this is vastly different to the results obtained here, where $36 \mathrm{cP}$ occurs when the temperature of the fluid is $35^{\circ} \mathrm{C}$. The discrepancy in values reported at low viscosities might be clarified through experimental limitations of the rotational viscometer.

Glyceline is formed using glycerol as the HBD. The data obtained from both methods, i.e. rotational viscometer and QCM, correlated extremely well (Fig. 2b), with a difference of $1 \mathrm{cP}$ at high temperatures and $\approx 30 \mathrm{cP}$ at room temperature. The rotational viscometer data ranges from $23.4 \mathrm{cP}$ at $85^{\circ} \mathrm{C}$ down to $345.4 \mathrm{cP}$ at $25^{\circ} \mathrm{C}$, whereas the $\mathrm{QCM}$ calculated viscosities range from $23.5 \mathrm{cP}$ at $85^{\circ} \mathrm{C}$ to $402.9 \mathrm{cP}$ at room temperature.

This data highlights the potential of QCM for the application as a viscometer. Literature values for the viscosity of glyceline are available [13], with the viscosity being reported as $259 \mathrm{cP}$ at $25^{\circ} \mathrm{C}$. This value is $86.4 \mathrm{cP}$ higher than the viscosity measured during this experiment. As all batches of DES are slightly different, it would be expected that the viscosities may not match exactly, however there must be a different explanation for this large discrepancy in viscosity.

Reline, formed with urea as the HBD, has a much higher viscosity at low temperatures than both ethaline and glyceline. At the higher temperatures, the two sets 
of data appear to give comparable results (Fig. 2c), with a difference of $18 \mathrm{cP}$ between the two at $85^{\circ} \mathrm{C}$. However, at $35^{\circ} \mathrm{C}$ the data sets begin to diverge quite drastically, ending with a difference of $57 \mathrm{cP}$ at $30^{\circ} \mathrm{C}$. Previous work [14] reports the viscosity of reline as being ca. $460 \mathrm{cP}$ at $30^{\circ} \mathrm{C}$, this is comparable with the $\mathrm{QCM}$ calculated viscosity reported here as $\approx 506 \mathrm{cP}$ at $30^{\circ} \mathrm{C}$, whereas the rotational viscometer reports a viscosity of $563 \mathrm{cP}$ at $30^{\circ} \mathrm{C}$. This leads to the conclusion that the QCM data reported here is more reliable than the rotational viscometer data, as it correlates far better with the previously reported viscosity values. This discrepancy between the two sets of data at low temperatures could be due to experimental limitations of the rotational viscometer.

Maline has a much higher viscosity at low temperatures than other DESs investigated here. Unlike ethaline, glyceline and reline even at the high temperatures, the two sets of data do not give comparable results (Fig. 2d), with a difference of $\approx 140 \mathrm{cP}$ between the two at $85^{\circ} \mathrm{C}$. However, at $35^{\circ} \mathrm{C}$ the data sets begin to diverge quite drastically, ending with a difference of more than $900 \mathrm{cP}$ at $35^{\circ} \mathrm{C}$. The viscosity values obtained from rotational viscometer (Fig. 2d) for maline show a dramatic decrease in the order of $500 \mathrm{cP}$ between 35 and $50^{\circ} \mathrm{C}$. After this point values of viscosity begin to level off and remain in the region of approximately 150-200 cP decreasing dramatically slowly after this point. This shows that as temperature increases, its effect on the viscosity of a fluid becomes less extreme. Finally, the data of maline shows significant changes, particularly before the addition of water. When the temperature is $65^{\circ} \mathrm{C}$ viscosity is $\approx 245 \mathrm{cP}$ and this value rapidly increases until cooling to $30^{\circ} \mathrm{C}$ when it becomes $\approx 1006 \mathrm{cP}$.

\subsection{Sequential water additions}

To determine whether there was a change in the properties of the DESs when water was added, a slightly steady decrease in viscosities were observed in ethaline, glyceline, and reline. Suggesting that, these liquids behave in a Newtonian manner, while maline behaves in a non-Newtonian manner. Newtonian fluids should have a constant viscosity with shear rate and this is clearly not the case with maline. A decrease in viscosity with shear rate means that at low rotation speed the interionic and intermolecular forces are large and resist the motion of the spindle. At higher rotation speeds the ions do not have sufficient time to reform their interactions and so the viscosity decreases.

Sequential additions of deionized water were added to the DESs, and the viscosity of the diluted solution was measured using the rotational viscometer. The data was collected using the same method that had been established for the pure DESs, the only difference being that the water was added prior to the DES being heated.

The same patterns were observed for the rest of the DESs after dilutions with slight variations and higher viscosities. In general, all the liquids have higher viscosity before the addition of water. Hence, each solution is more viscose without $\mathrm{H}_{2} \mathrm{O}$, in a way that in most measurements they are completely separated with diluted fluids.

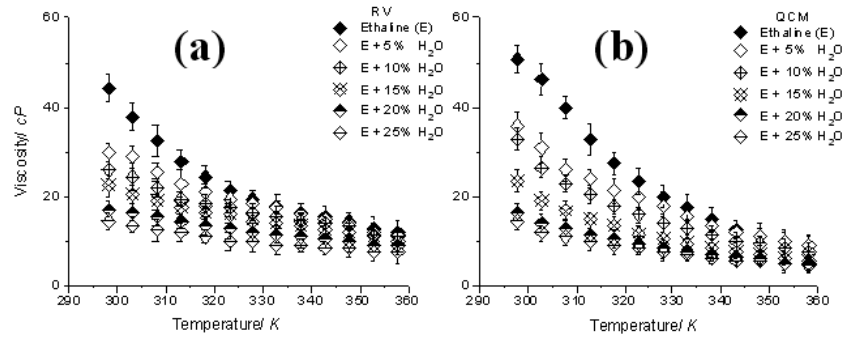

Fig. 3. Viscosity of ethaline using (a) RV, (b) QCM with water additions, $5 \%-25 \%$ by volume as a function of temperature.

As with the pure DESs, the viscosity remains a function of temperature even as a diluent is added to the DES. The viscosity of ethaline decreases with the addition of water (Fig. 3), with the most pronounced impact occurring at the lowest temperatures. Using rotational viscometer at $25^{\circ} \mathrm{C}$ there is a $30 \mathrm{cP}$ difference in viscosity between the pure ethaline and the ethaline containing $25 \% \mathrm{H}_{2} \mathrm{O}$, compared to only a $4.5 \mathrm{cP}$ difference at $85^{\circ} \mathrm{C}$. The drop in viscosity between the dilutions does not appear to be a steady decrease (Fig. 3a), while within the QCM technique at the same temperatures the differences are 36 and $4.6 \mathrm{cP}$ at $25^{\circ} \mathrm{C}$ and $85^{\circ} \mathrm{C}$, respectively (Fig. 3b). These results clarify that the QCM technique can obtain viscosity values that correspond to the values determined by the current method of viscosity measurement, rotational viscometer.

This trend is unexpected, as it would be assumed that the dilutions would produce a constant decrease in viscosity as the dilution increased. A possible explanation could be that some of the water evaporated off during the reheating of the DES, meaning that there was not the correct volume of water present in the DES during the experiment.

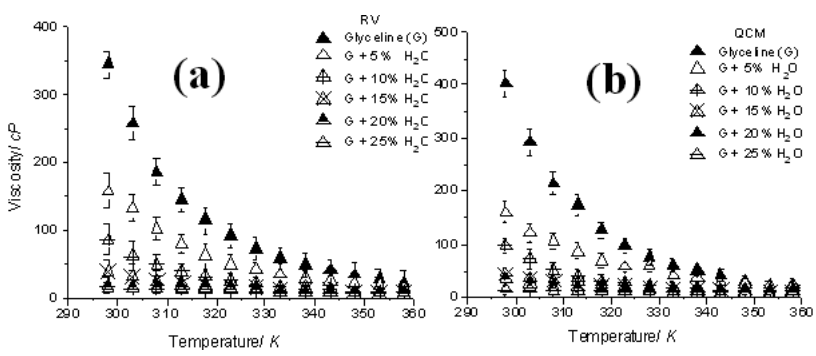

Fig. 4. As in Fig. 3, but for glyceline.

In the case of glyceline all liquids possess steadily increasing viscosity (Fig. 4) when they cooled down except for two measurements; including glyceline before dilution and $5 \%$ added water. Hence the curves of the last four liquids, glyceline $10 \%$, glyceline $15 \%$, glyceline $20 \%$ and 
glyceline $25 \%$, are quite close to each other meaning differences are difficult to discern. On the other hand, glyceline and glyceline with $5 \% \mathrm{H}_{2} \mathrm{O}$ viscosities' are raised and their difference is easily observable. A significant change in the viscosity of glyceline occurs in the temperature range between 45 and $25^{\circ} \mathrm{C}$. When the temperature is at $85^{\circ} \mathrm{C}$ until it cools to $50^{\circ} \mathrm{C}$ viscosities are under $100 \mathrm{cP}$, after these range rapid changes are observed. The range varies from just above $100 \mathrm{cP}$ to $346.13 \mathrm{cP}$ when the solution cools to room temperature.

Similar patterns are shown for reline in comparison with glyceline and its water ratios. This similarity is easier to observe when the temperature range is $75-50^{\circ} \mathrm{C}$ (Fig. 5). At $75^{\circ} \mathrm{C}$ using rotational viscometer the viscosities of glyceline are $21.90,11.52,10.52,8.80$, and $7.87 \mathrm{cP}$ for $5 \%, 10 \%, 15 \%, 20 \%$, and $25 \%$ water, respectively. Likewise, the values for the viscosities of reline are 41.39, $18.88,12.4,8.82,7.74$, and $6.64 \mathrm{cP}$ for reline itself and its water proportions (means one for reline without water and then 5 percentages of water corresponding to them). Both DESs and their diluted solutions have viscosities under $50 \mathrm{cP}$.

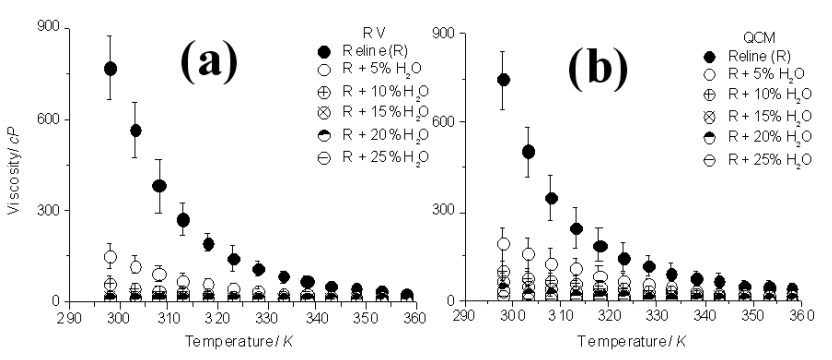

Fig. 5. As in Fig. 3, but for reline.

Finally, the data of maline shows significant changes, particularly before the addition of water (Fig. 6). When the temperature is $65^{\circ} \mathrm{C}$ using rotational viscometer, viscosity is $184.58 \mathrm{cP}$ and this value rapidly increases until cooling to $30^{\circ} \mathrm{C}$ when it becomes $1001.69 \mathrm{cP}$. While, through using QCM, taking into consideration the same temperature values, the viscosities of maline are $11.56 \mathrm{cP}$ and $48.12 \mathrm{cP}$ for $65^{\circ} \mathrm{C}$ and $30^{\circ} \mathrm{C}$, respectively. Maline shows great differences in viscosity values for both methods. Moreover, these data are different in comparison to other DESs.

The viscosity of maline is an important aspect shown in the graph which requires discussion. It seems entirely diverse as it was expected. Maline is the most viscose liquid when it is compared with other DES. The reason for this is the properties of maline. It was prepared by mixing the choline chloride with malonic acid, and then highly volatile liquid was obtained. Vaporisation occurs particularly when it is heated to high temperatures because it has a hydrogen bond which is easily released from the liquid into air. Another reason is the existence of extremely high bubbles in the fluid which are again due to the hydrogen bonds. As was mentioned in the experimental section, during the setup of the QCM the cells crystal must be protected from air bubbles, whereas it was impossible to protect this from bubbles in maline where they were continually being produced.

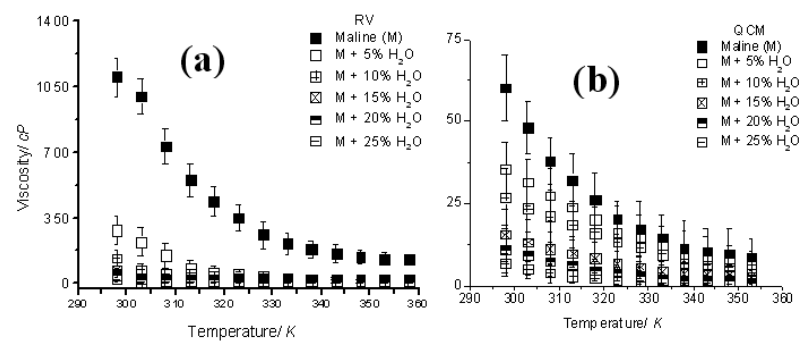

Fig. 6. As in Fig. 3, but for maline.

On the other hand, higher variations can be seen in comparison to solutions after the dilution with water. This difference in maline before dilution is clearly seen in Fig. 6, in which it is entirely isolated from solutions following the water dilution process. Similarly, when the viscosity of liquids of maline tested after the addition of water is compared with other DES, small variations are recorded. The main factor contributing to this significant difference may be the chemical composition of maline due to its main component, i.e. malonic acid. The acidity of malonic acid is high which causes a large amount of bubbles. These bubbles can form a barrier between the liquid and the quartz crystal. Preliminary data suggests that the viscosity of maline behaves as non-Newtonian fluid at low temperature and behaves as Newtonian fluid at relatively high temperature. Also, adding water from $5-25 \mathrm{wt} \%$ will change the non-Newtonian behavior to the Newtonian.

\section{Conclusion}

Due to their high viscosities DESs are required to be investigated using newly developed techniques. In order to obtain accurate information concerning physical and rheological properties, QCM has been applied. QCM is a novel instrument with high accuracy. It is quick, simple, requires a small sample volume which reduces costs, particularly for expensive compounds, and for highly viscous fluids.

This study was performed to assess the potential of QCM in measuring the viscosity of a series of DES with different properties. For this reason, it was employed alongside with the rotational viscometer to assess its accuracy. For most cases comparable data were observed except for maline. It was expected that maline would be the most viscous fluid in the series. The conventional viscometer proved this but QCM did not support these expectations. Perhaps due to the high acidity of maline, this led to a large number of bubbles. Alongside the high sensitivity of the QCM, these factors contributed to the difference. Increases in viscosity were recorded as a consequence of decrease of the fluid temperature. 
Another attempt of this investigation was adding water to the DES, which led to decreases in viscosity. Through increase of the amount of the diluent a decrease in fluid viscosity was produced. In maline eutectic the addition of water decreases the change of viscosity with shear rate but it still decreases showing that non-Newtonian behavior exists.

\section{Acknowledgments}

The authors would like to thank the Materials Center, Leicester University, Leicester, UK.

\section{References}

[1] G. Sauerbrey, Z. Phys. 155, 206 (1959).

[2] V.M. Mecea, Anal. Lett. 38, 753 (2005).

[3] K.K. Kanazawa, J.G. Gordon, Anal. Chim. Acta 175, 99 (1985).

[4] K.A. Marx, Biomacromolecules 4, 1099 (2003).
[5] M. Rodahl, F. Höök, A. Krozer, P. Brzezinski, B. Kasemo, Rev. Sci. Instrum. 66, 3924 (1995).

[6] S. Bruckenstein, M. Shay, Electrochim. Acta 30, 1295 (1985).

[7] S.J. Martin, V.E. Granstaff, G.C. Frye, Anal. Chem. 63, 2272 (1991).

[8] D.A. Buttry, M.D Ward, Chem. Rev. 92, 1355 (1992).

[9] M.D. Ward, D.A. Buttry, Science 249, 1000 (1990).

[10] Electrodeposition from Ionic Liquids, Eds. F. Endres, D. MacFarlane, A. Abbott, Wiley, Germany 2008.

[11] A.R. Aitkenhead, G. Smith, D.J. Rowbotham, Textbook of Anaesthesia, Churchill Livingstone Elsevier, London 2007.

[12] A.P. Abbott, R.C. Harris, K.S. Ryder, J. Phys. Chem. B 111, 4910 (2007)

[13] A.P. Abbott, R.C. Harris, K.S. Ryder, C. D'Agostino, L.F. Gladden, M.D. Mantle, Green Chem. 13, 82 (2011).

[14] A.P. Abbott, G. Capper, D.L. Davies, R.K. Rasheed, V. Tambyrajah, Chem. Commun. 2003, 70 (2003). 